\title{
Economic and Humanistic Burden of Cervical Cancer in the United States: Results from a Nationally Representative Survey
}

\author{
Ruchit Shah, PhD, ${ }^{1}$ Chizoba Nwankwo, $\mathrm{PhD},{ }^{2}$ \\ Youngmin Kwon, BA, and Shelby L. Corman, PharmD, MS, BCPS ${ }^{1}$
}

\begin{abstract}
Objective: To measure the economic and humanistic burden of cervical cancer in the United States.

Materials and Methods: This was a retrospective analysis of Medical Expenditure Panel Survey data (20062015). Cervical cancer cases were identified using International Classification of Diseases, Ninth Revision, Clinical Modification code "180" or clinical classification software code "26". The control group included women without any cancer diagnosis. Study outcomes included health care resource use (institutional inpatient and outpatient, emergency room, and physician office visits), costs, limitations in activities of daily living, and quality of life (general health status, 12-Item Short Form Health Survey [SF-12] physical and mental component summary [MCS], EuroQol-5D and Short-Form Six-Dimension health utility, and Patient Health Questionnaire2 depression severity). Generalized linear models, controlling for sociodemographic and clinical covariates, were conducted to compare outcomes between cases and controls.

Results: The analytic cohort included 275,246 cervical cancer cases and 146,061,609 noncancer controls. Cases were significantly older (mean age [years]: 42.03 vs. 36.98 ) and had a higher Charlson comorbidity burden (mean score: 1.06 vs. 0.46) versus controls. Multivariate analyses suggested that compared to controls, cancer cases had significantly higher costs: institutional outpatient (\$1,610 vs. $\$ 502)$, physician visit $(\$ 2,422$ vs. $\$ 1,321$ ), and total health care $(\$ 10,031$ vs. $\$ 4,913)$. Cases were 1.99 (odds ratio [OR]: $1.991 ; 95 \%$ confidence interval [CI]: $1.23-3.22$ ) and 2.56 (OR: $2.562 ; 95 \%$ CI: 1.78-3.68) times as likely to report activity limitations and poor general health versus controls. Cervical cancer patients had significantly lower SF-12 physical and MCS score, health utility, and higher depression severity.

Conclusions: Cervical cancer is associated with significant economic burden, activity limitations, and quality of life impairment among ambulatory women in the United States.
\end{abstract}

Keywords: cervical cancer, economic burden, quality of life, activity limitations, survey

\section{Introduction}

A N ESTIMATED 13,170 women in the United States will be diagnosed with cervical cancer in 2019 , with 4,210 deaths from the disease. Disease burden of cervical cancer is well described in terms of mortality and life years lost, ${ }^{1}$ but little is known about the direct economic burden of the disease.

Health care costs in Medicare-insured cervical cancer survivors in Texas totaled more than $\$ 15$ million in
2009 alone. Cost per cervical cancer survivor was $\$ 9,827$ compared to $\$ 6,142$ for the average Medicare enrollee after adjustment for age, sex, and race. ${ }^{2}$ In a commercially insured population in Texas between 2001 and 2014, health care costs in the first and second years after diagnosis were $\$ 50,846$ and $\$ 27,656$ higher, respectively, in cervical cancer cases compared to controls. ${ }^{3}$ National estimates of cervical cancer costs were last published in 2008 (reported in 2003 US dollars), at which

\footnotetext{
${ }^{1}$ Pharmerit International, Bethesda, Maryland.

${ }^{2}$ Merck \& Co., Inc., Kenilworth, New Jersey.
}

(C) Ruchit Shah et al. 2020; Published by Mary Ann Liebert, Inc. This Open Access article is distributed under the terms of the Creative Commons Attribution Noncommercial License (http://creativecommons.org/licenses/by-nc/4.0/) which permits any noncommercial use, distribution, and reproduction in any medium, provided the original author(s) and the source are cited. 
time mortality adjusted 4-year health care costs were $\$ 29,649$. $^{4}$ Recent country-level estimates of economic burden are lacking.

Disease burden from a humanistic perspective includes health-related quality of life (HRQoL) and activities of daily living among patients and their caregivers. Quality of life in cervical cancer patients has been described in a number of clinical trials and longitudinal studies, ${ }^{5}$ but there are no data benchmarking quality of life in cervical cancer patients to that of women without cancer. In addition, most studies that have been conducted employed only a limited number of quality of life measures such as Functional Assessment of Cancer Therapy: General and/or The European Organization for Research and Treatment of Cancer (EORTC) core quality of life questionnaire. However, these studies did not evaluate impact of cervical cancer on functional limitations and activities of daily living. ${ }^{6}$ Moreover, there are no studies that have evaluated the work productivity losses associated with cervical cancer.

An accurate understanding of cancer burden is needed to direct and formulate health care interventions and strategies, and to allow for optimal allocations of scarce health care resources. ${ }^{7}$ Therefore, the objective of this study was to estimate the direct and indirect economic and humanistic burden of cervical cancer patients compared to noncancer controls in a nationally representative sample of the United States population.

\section{Materials and Methods}

\section{Study design/data source}

This was a retrospective, cross-sectional study using data from the Medical Expenditure Panel Survey (MEPS) sponsored by the Agency for Healthcare Research and Quality, collected for the years 2006-2015. MEPS is a nationally representative survey of civilian, noninstitutionalized population in the United States. The survey collects data and provides detailed information on demographic and clinical characteristics, health status, and health care utilization and expenditures. ${ }^{8}$ This study used the Household Component of MEPS, which provides the data from each person in individual households, supplemented with data from their medical providers. ${ }^{9}$ Because MEPS contains de-identified data that are publicly available, no review by an Institutional Review Board was deemed necessary.

The MEPS household component is designed to produce national level estimates of the health care use, expenditures, sources of payment, and insurance that are generalizable to a population of noninstitutionalized United States adults. The sample selection for the MEPS survey includes stratification, clustering, several stages of selection, and disproportionate sampling. Therefore, the MEPS data include three weighting variables namely: the person weight (PERWT), stratum (VARSTR), and PSU (VARPSU). These weighting variables also reflect adjustment for survey nonresponse.

\section{Patient cohort}

The study included two cohorts: women with cervical cancer and noncancer controls. Cervical cancer patients were identified in the data by using the Clinical Classification Code (CCS) of "026" and International Classification of Diseases, Ninth Revision, Clinical Modification (ICD-9 CM) code of
"180 in the following MEPS event files: hospital inpatient stays, institutional outpatient visits, emergency room visits, office-based visits, and full-year consolidated files. Women without cancer-related CCS (011-047) and ICD-9 CM codes (140-239) were designated as noncancer controls.

\section{Demographic variables/comorbidities}

Data on key demographic variables and comorbidities were obtained from the MEPS full-year consolidated files and medical condition files. Demographic variables extracted included age, body mass index, census region, education, employment status, family income, insurance status, and race/ethnicity. Comorbidities were identified in the medical condition and event files by using the CCS and ICD-9 CM codes as per the adapted Charlson Comorbidity Index (CCI) by D'Hoore et al., which predicts a patient's 1-year mortality risk based on the presence of 22 comorbid conditions. ${ }^{10}$

\section{Direct/indirect economic burden of cervical cancer}

Total (all-cause) costs were calculated by summing up the costs associated with any health care services in the MEPS event files. Number of prescriptions and inpatient, hospital outpatient, office, and emergency room visits were calculated to determine all-cause health care resource use. Indirect costs were calculated based on the answer to the following question: response to the question "how many days, did you miss a half day or more from work/school due to physical illness or injury, or a mental or emotional problem?" The total number of days of work/school missed were reported as a measure of absenteeism. All costs were converted to 2017 United States dollars, adjusted for inflation by using Consumer Price Index for medical care.

\section{Humanistic burden of cervical cancer}

Humanistic burden of cervical cancer was measured in terms of HRQoL, self-reported depression scores, limitations in activities of daily living, and health utility. In MEPS, the participants are asked to rank their perceived general/mental health status on a five-category scale: 1, Excellent; 2, Very good; 3, Good; 4, Fair; or 5, Poor. For all analyses the perceived mental health and general health status variable were dichotomized into good health (containing responses of excellent, very good, and good health) and poor health (containing responses of fair and poor health). ${ }^{11-14}$ In addition, the following quality of life questionnaires were administered, and their data were available in the full-year consolidated files: 12-Item Short Form Health Survey Version 2 (SF-12 v2), ${ }^{15}$ reported as physical component summary (PCS) and mental component summary (MCS) scores (ranging from 0 to 100 , with higher scores indicating better physical and mental HRQoL, respectively), and Patient Health Questionnaire-2 (PHQ-2), which measures frequency of depressed mood (ranging from 0 to 6 ; higher scores represent greater predisposition toward depression). Based on the response to individual items on the SF-12 v2, Short-Form Six-Dimension (SF6D), and EuroQol-5D (EQ-5D) utilities were calculated by using previously published utilities value set and algorithms. ${ }^{16,17}$ Lastly, the survey also inquired participants about functional limitations (yes/no) in the following domains: physical functioning, daily activities, social/recreational limitations, and cognitive limitations. 
Table 1. Demographic, Clinical Characteristics, and Self-Reported Health Status in Patients With Versus Without Cervical Cancer

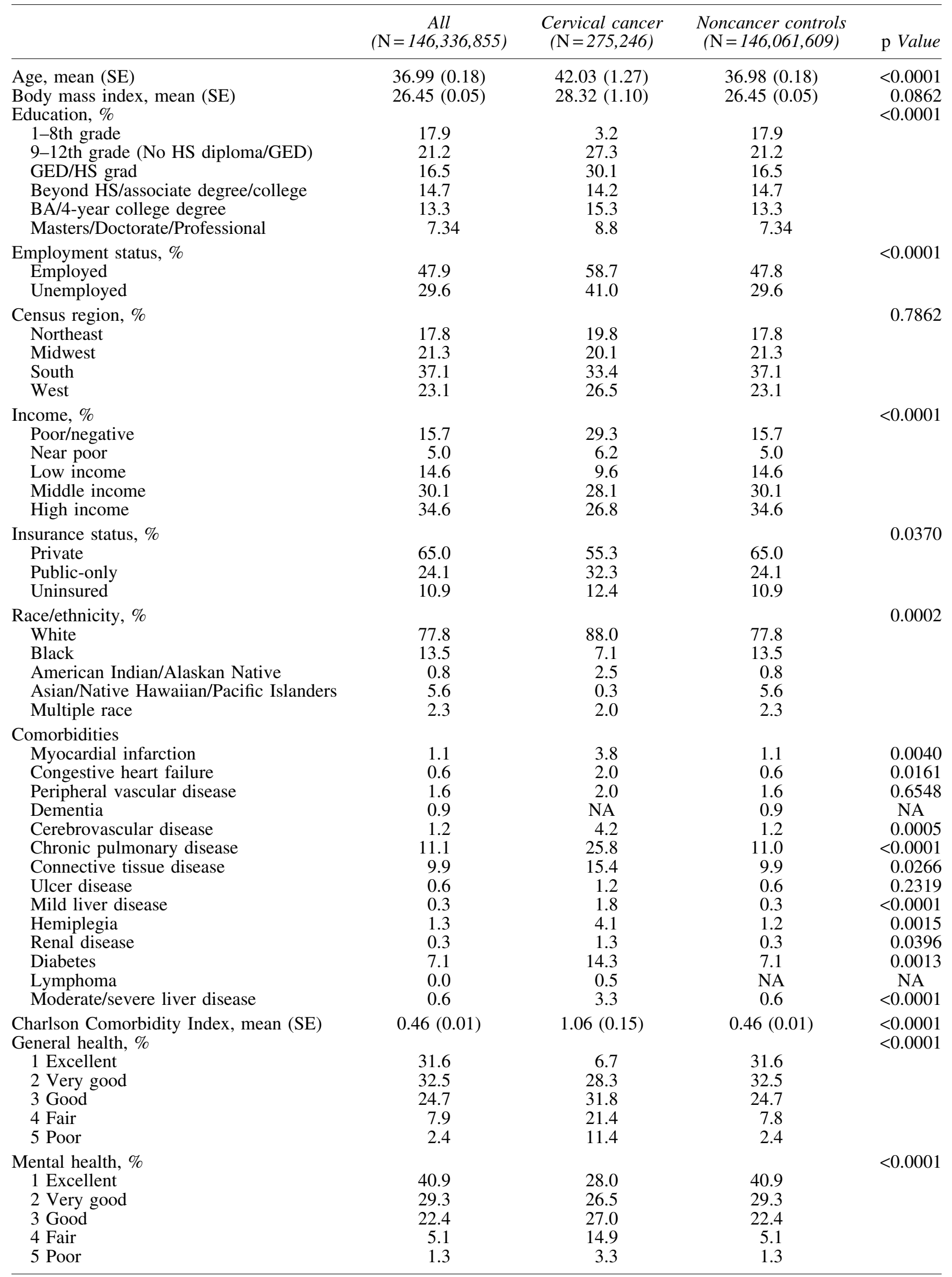

SE, standard error; HS, high school; GED, general education development; BA, Bachelor's degree; NA, not available. 


\section{Statistical analysis}

Descriptive statistics (mean or proportion \pm standard error [SE]) were generated to characterize and compare women with cervical cancer and noncancer controls. Bivariate analyses were conducted using chi-square tests for categorical variables and $t$-tests for continuous variables. For multivariate analyses, logistic regressions were used to evaluate the relationship between cervical cancer, and functional limitations and health status. Linear regressions were used to compare SF12 v2 PCS score, MCS score, and PHQ-2 depression severity between cervical cancer and noncancer controls. Beta regressions were used for the SF-6D and EQ-5D health utility outcomes. Generalized linear models with gamma distribution/log link and Poisson distribution were used to compare cervical cancer cases and noncancer controls in terms of health care costs and health care resource use, respectively. Predicted adjusted cost and resource use estimates were obtained from these generalized linear models. Covariates included in these models were: cancer status (cervical cancer or control), age, race/ethnicity (White, Black, American Indian/Alaskan Native, Asian/Native Hawaiian/Pacific Islander), education (18th grade, 9-12th grade [no high school diploma/general education diploma], general education diploma/high school grad, beyond high school/associate degree/college, Bachelor of Arts/4-year college degree, or masters/doctoral/professional, as provided by MEPS), census region (Northeast, Midwest, South, or West), insurance (private, public only, or uninsured, as provided in MEPS), body mass index, CCI, family income (poor/negative, near poor, low income, middle income, or high income, as provided by MEPS), and employment status (employed or unemployed).

All analyses were carried out in SAS v9.4 and STATA 14 using survey procedures to produce weighted estimates and derive associated SEs. To account for this complex survey design, all analyses were performed while accounting for stratification and clustering in MEPS to produce nationally representative means and proportions. Pooled weights were used as a weighting variable. It is not appropriate to assume simple random sampling and perform statistical analysis using standard analytic procedures.

\section{Results}

\section{Study cohort characteristics}

In the analytic cohort, there were 275,246 cases of cervical cancer and 146,061,609 noncancer controls (Table 1). Cervical cancer cases were significantly older (mean age: 42.03 [1.3] vs. $36.98[0.2] ; p<0.0001)$ and had a higher comorbidity burden (CCI: 1.06 [0.2] vs. 0.46 [0.01]; $p<0.0001$ ). Cervical cancer cases were more likely to report "poor" general and mental health $(p<0.0001)$. Moreover, cervical cancer cases had significantly higher proportions of those with high school degrees (including general educational degrees), bachelor's, master's, doctorate, and professional degrees $(p<0.0001)$. The cases and controls also differed significantly on race/ ethnicity groups distribution, with a higher representation of whites and American Indian/Alaskan natives among cervical cancer cases $(p=0.0002)$.

\section{Economic burden of cervical cancer}

Results from the generalized linear model indicated that cervical cancer patients incurred higher annual health care expenses (Figs. 1 and 2). Specifically, cervical cancer patients incurred significantly higher total costs $(\$ 10,031$ vs. $\$ 4,913$; $p=0.0120)$, office-based costs $(\$ 2,422$ vs. $\$ 1,321 ; p=0.0003)$, and institutional outpatient costs $(\$ 1,610$ vs. $\$ 502 ; p=0.0282)$. The results also suggested that cervical cancer patients had significantly higher office visits compared to the controls (mean: 8.87 vs. $6.41 ; p=0.005$ ). There were no significant differences between the two groups in terms of missed work days per year (mean: 1.83 vs. $0.93 ; p=0.132$ ).

\section{Humanistic burden of cervical cancer}

In the unadjusted analyses, cervical cancer cases had significant quality of life impairment compared to the noncancer controls (Table 2). Cervical cancer cases were more likely than controls to report functional limitations in all domains (all $p<0.05$ ). All quality of life scores, except for SF-6D utility, indicate worsened disability among cervical cancer cases than the controls (lower PCS/MCS scores, higher PHQ2 scores, and EQ-5D utility).
FIG. 1. Adjusted per person per year mean health care resource use among patients with versus without cervical cancer. The following covariates were included in the model to predict adjusted health care resource use/costs: cancer status, age, race, education, region, insurance, body mass index, Charlson Comorbidity Index, family income, employment status.

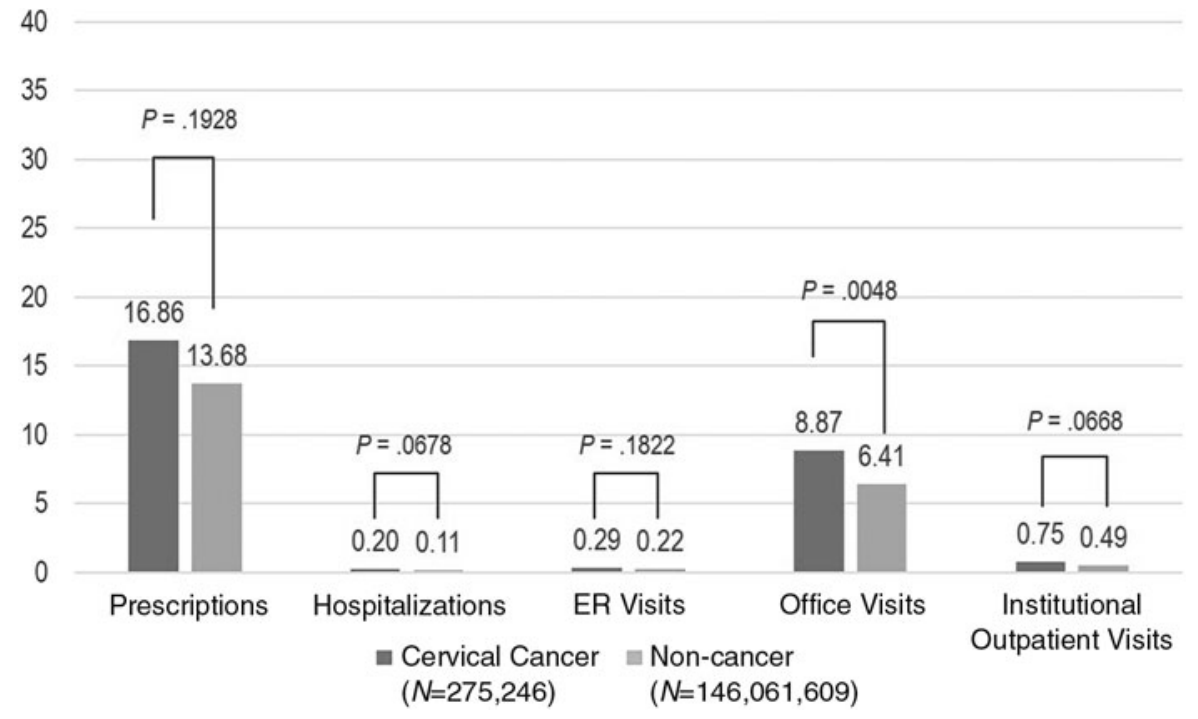




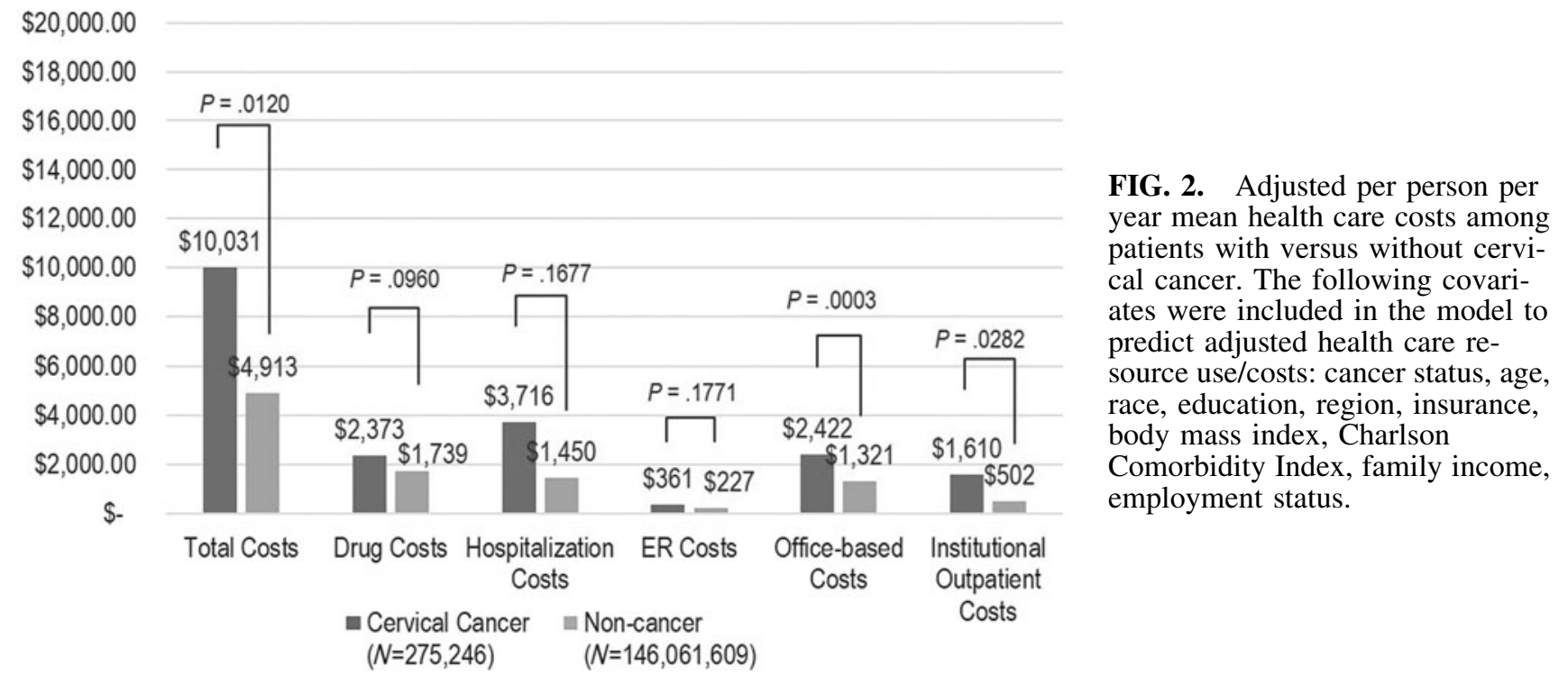

In the multivariate analyses, cervical cancer patients were 1.99 (odds ratio [OR]: $1.99 ; 95 \%$ confidence interval [CI]: 1.23-3.22) and 2.56 (OR: 2.56; 95\% CI: 1.78-3.68) times as likely to have activity limitations and poor general health when compared to controls (Table 3). Moreover, they had a significantly lower SF-12 PCS (beta [SE]: -2.586 [0.995]; $p<0.05)$, MCS $(-2.224$ [0.911]; $p<0.05)$, EQ-5D utility $(-0.427$ [0.106]; $p<0.0001)$, and higher PHQ-2 depression severity score $(0.356[0.130] ; p<0.05)$ than noncancer controls.

\section{Discussion}

The incidence of cervical cancer has decreased by more than half since the 1950s when Pap smear screening was incorporated into practice and allowed for earlier detection of disease. ${ }^{18}$ However, incidence and mortality have remained stable in more recent decades, and cervical cancer remains an important threat to public health due to low uptake of human papillomavirus vaccination and cervical cancer screening. ${ }^{19}$ To allocate health care resources optimally, an accurate understanding of cancer burden is needed. ${ }^{7}$ The substantial patient-level economic burden and worse quality of life among patients with cervical cancer compared to controls demonstrated that there is still work to be done in reducing the burden of cervical cancer to patients, the health care system, and society.

In the current analysis, we found that cervical cancer patients had significantly higher total all-cause health care costs, institutional outpatient, and physician visit costs compared to noncancer controls. Specifically, having cervical cancer resulted in significantly higher total health care costs $(\$ 10,031$ vs. $\$ 4,913 ; p=0.0120)$, office-based costs $(\$ 2,422$ vs. $\$ 1,321 ; p=0.0003)$, and institutional outpatient costs $(\$ 1,610$ vs. $\$ 502 ; p=0.0282)$. Estimates of direct and indirect health care costs associated with cervical cancer in the literature have been rare. Eberth et al. reported the economic burden of cervical cancer in Texas after analyzing regional cancer registry data and found substantial costs among cervical cancer patients. ${ }^{2}$ Specifically, cervical cancer survivors over the age of 65 spent average of 12.1 and 5.1 days in inpatient and outpatient care, respectively, with $\$ 11$ million in hospitalization costs and $\$ 3$ million in outpatient costs in 2009. Lairson et al. analyzed commercially insured cervical cancer patients in Texas and observed first-year medical costs of $\$ 60,828$ for cervical cancer cases and $\$ 9,982$ for controls. ${ }^{3}$ A study by Insinga et al. estimated health care resource use and costs of three cancers, including cervical cancer, among commercially insured (with or without Medicare or Medicaid dual coverage) patients enrolled in a large

Table 2. Quality of Life in Patients With Versus Without Cervical Cancer

\begin{tabular}{lccrr}
\hline & $\begin{array}{c}\text { All } \\
(\mathrm{N}=146,336,855)\end{array}$ & $\begin{array}{c}\text { Cervical cancer } \\
(\mathrm{N}=275,246)\end{array}$ & $\begin{array}{c}\text { Noncancer } \\
(\mathrm{N}=146,061,609)\end{array}$ & $\mathrm{p}$ Value \\
\hline Physical limitations, \% & 10.2 & 18.7 & 10.2 & $<0.0001$ \\
Activity limitations, \% & 7.5 & 21.1 & 7.5 & $<0.0001$ \\
Social limitations, \% & 4.3 & 8.2 & 4.3 & 0.0018 \\
Cognitive limitations, $\%$ & 3.9 & 9.3 & 3.8 & $<0.0001$ \\
SF-12 PCS, mean (SE) & $49.19(0.08)$ & $45.36(1.17)$ & $49.20(0.08)$ & 0.0011 \\
SF-12 MCS, mean (SE) & $50.39(0.06)$ & $46.16(0.99)$ & $50.40(0.06)$ & $<0.0001$ \\
PHQ-2 score, mean (SE) & $0.74(0.01)$ & $1.33(0.14)$ & $0.74(0.01)$ & $<0.0001$ \\
SF-6D utility score, mean (SE) & $0.56(0.00)$ & $0.57(0.01)$ & $0.56(0.00)$ & 0.0157 \\
EQ5D utility score, mean (SE) & $0.86(0.00)$ & $0.78(0.01)$ & $0.86(0.00)$ & $<0.0001$ \\
\hline
\end{tabular}

SF-12, 12-Item Short Form Health Survey Version 2; PCS, physical component summary; SE, standard error; MCS, mental component summary; PHQ-2, Patient Health Questionnaire-2; SF-6D; Short-Form Six-Dimension health utility; EQ5D, EuroQol-5D. 
Table 3. Multivariate Analyses to Assess

Associations Between Cervical Cancer AND Patient-Reported Outcomes

\begin{tabular}{lcr}
\hline & Odds ratio & $\mathrm{p}$ Value \\
\hline Activity limitation & $1.99(1.23-3.22)$ & 0.0051 \\
Physical limitation & $1.32(0.70-2.49)$ & 0.3990 \\
Social limitation & $0.92(0.46-1.82)$ & 0.8004 \\
Cognitive limitation $^{\text {Mental health }}{ }^{\mathrm{a}}$ & $1.33(0.76-2.31)$ & 0.3220 \\
General health $^{\mathrm{a}}$ & $1.40(0.93-2.13)$ & 0.1110 \\
& $2.56(1.78-3.68)$ & $<0.0001$ \\
\hline & Beta $($ SE) & $\mathrm{p}$ Value \\
MCS score & $-2.586(0.995)$ & 0.0096 \\
PCS score & $-2.224(0.911)$ & 0.0150 \\
PHQ2 score & $0.356(0.130)$ & 0.0066 \\
EQ5D utility & $-0.427(0.106)$ & $<0.0001$ \\
SF-6D utility & $0.035(0.026)$ & 0.1740 \\
\hline
\end{tabular}

The multivariate analyses adjusted for age, race/ethnicity, education, census region, insurance status, body mass index, Charlson Comorbidity Index, family income, and employment status.

${ }^{a}$ The odds ratios indicate the probability of having worse perceived mental/general health status (fair/poor/negative vs. excellent/very good).

United States health plan. ${ }^{4}$ They found cumulative 4-year health care costs of $\$ 29,649$ after adjusting for mortality. However, these studies have limitations in external validity/generalizability as the study populations were limited to the adult cancer survivors in Texas, ${ }^{2,3}$ and/or the cancer survivors in private insurance settings only. ${ }^{3,4} \mathrm{Al}$ though, more generalizable, the cost estimates for cervical cancer from our analysis are lower than those from previous literature. This is because the estimates reported herein are the annual estimates of prevalent cases of cervical cancer. Prevalent costs of cases are considerably higher immediately after the diagnosis or at the end of life. ${ }^{20,21}$ As this was a cross-sectional analysis, it was not possible to determine diagnosis dates for the study population or follow them up until the end of life. Also, it was not possible to stratify the estimates by stage due to lack of staging information in MEPS dataset. Therefore, estimates here cannot be directly compared with those found in studies previously mentioned.

In cervical cancer, there are very few United States-based studies that evaluated quality of life and humanistic burden. A study by Eberth et al. showed that women with history of cervical cancer in Texas had worse overall, physical and mental health. ${ }^{2}$ Pfaendler et al. ${ }^{22}$ summarized existing literature on quality of life studies (both United States and non-United States countries) of cervical cancer patients and interviews of cancer survivors regarding social support. They concluded that the while data are mixed and inconclusive, cancer survivors' quality of life differed than that of baseline controls. Our analysis is one of the first to quantify the humanistic burden of cervical cancer patients on a national level, which suggested worsened quality of life among cervical cancer patients.

Furthermore, the review of the literature on quality of life studies reveals that most studies (including the ones not based in the United States) used cancer-specific questionnaires such as Functional Assessment of Cancer Therapy: General and The EORTC core quality of life questionnaire. ${ }^{5,6}$ While the quality of life measurements evaluated in this study are ge- neric, they can still provide important insights into the wellbeing of cervical cancer patients, especially when supplanted with results incorporating more disease-specific evidence. ${ }^{23}$ The adjusted differences in SF-12 scores between cervical cancer and noncancer controls were $-2.6(1.0)$ and -2.2 (0.9) for MCS score and PCS score, respectively. Such differences are clinically important, based on the minimally important difference of 2 points. ${ }^{24}$ The larger difference in MCS between the cancer group and the controls than the difference in PCS suggest the need for increased awareness and focus on the mental health issue relating to cervical cancer.

Lastly, the quality of life of cervical cancer patients were summarized using two sets of health utility measures: EQ-5D and SF-6D. Our estimates of EQ-5D utilities were generally within the range of population estimates in other countries (between 0.77 and 0.85 ). ${ }^{25,26}$ Similar to these studies, cervical cancer patients in our study had significantly lower EQ5D utilities compared to controls ( 0.86 vs. $0.78 ; p<0.001)$. It is interesting that the SF-6D utility difference was not in the expected direction, as the cervical cancer group exhibited higher SF-6D utility compared to the controls, while EQ-5D utility difference was in the expected direction. One potential explanation for such a finding is the difference between EQ$5 \mathrm{D}$ and SF-6D instruments in measuring HRQoL. ${ }^{27}$ The EQ$5 \mathrm{D}$ tend to generate higher utility scores when compared to the SF-6D. This is especially true in healthy population or patients with mild conditions/symptoms. ${ }^{28}$ As such, in United States general population, EQ-5D may be more suitable to evaluate quality of life in patients with more morbidities due to the higher ceiling effect. ${ }^{29}$ Moreover, the change in SF-6D can also be underestimated because of a narrower range of utility scores (given that the SF-6D has a high lower boundary of 0.3), which can explain why no significant differences were detected in our sample. ${ }^{27}$ Overall, the difference in EQ-5D and SF-6D found in our analysis is consistent with the prior studies and the EQ-5D may be a better instrument at detecting quality of life differences between these two groups.

There are certain limitations to the study that must be acknowledged. First, the study employed a cross-sectional design, and therefore, it is not possible to establish causal inferences between cervical cancer and health care costs. In addition, the participants in MEPS are not systematically queried about their diagnosis, and only "treated prevalence" of cervical cancer cases are reported. ${ }^{30}$ Second, there is an issue of misclassification related to using CCS and the threedigit ICD-9 CM codes, which are broader categories of cancer that are less precise. Third, there is no information on time since diagnosis or disease stage. Future studies are needed to assess the burden of cervical cancer longitudinally, evaluating the relationship between disease staging, progression, and treatment on economic burden and quality of life. Finally, the analysis did not control for the human immune-deficiency virus (HIV) status of each patient. Given the elevated incidence of cervical cancer among patients with HIV ${ }^{31}$ future studies must account for this relationship while determining the burden of cervical cancer.

\section{Conclusions}

This study identified substantial patient-level economic burden; physical, activity, and cognitive limitations; and impaired quality of life in cervical cancer patients compared 
to controls. These results support continued investment in cervical cancer prevention and development of treatments in order to optimize economic and quality of life outcomes.

\section{Acknowledgments}

The authors would like to thank the following Pharmerit project team members for their contributions to the research, and critical review: Rachel Shah, Sophie Roberts.

\section{Author Disclosure Statement}

R.S., Y.K., and S.L.C. are employees of Pharmerit International, which received research funding from Merck and Co., Inc. for this study. C.N. is an employee of Merck and Co., Inc and owns Merck and Co. stock.

\section{Funding Information}

Pharmerit International is a HEOR consulting firm which directly received research funding from Merck and Co., Inc. for conducting this research.

\section{References}

1. National Cancer Institute. SEER cancer statistics review, 1975-2012, 2015. Available at: https://seer.cancer.gov/ archive/csr/1975_2012/ Accessed March 21, 2019.

2. Eberth JM, Prarelkar P, Nguyen H, et al. The human and economic burden of cervical cancer in Texas. Tex Public Health J 2013;65:51-55.

3. Lairson DR, Fu S, Chan W, et al. Mean direct medical care costs associated with cervical cancer for commercially insured patients in Texas. Gynecol Oncol 2017;145:108-113.

4. Insinga RP, Ye X, Singhal PK, et al. Healthcare resource use and costs associated with cervical, vaginal and vulvar cancers in a large U.S. health plan. Gynecol Oncol 2008; 111:188-196.

5. Tax C, Steenbergen ME, Zusterzeel PLM, et al. Measuring health-related quality of life in cervical cancer patients: A systematic review of the most used questionnaires and their validity. BMC Med Res Methodol 2017;17:15.

6. Vistad I, Fossa SD, Dahl AA. A critical review of patientrated quality of life studies of long-term survivors of cervical cancer. Gynecol Oncol 2006;102:563-572.

7. Jo C. Cost-of-illness studies: Concepts, scopes, and methods. Clin Mol Hepatol 2014;20:327-337.

8. Agency for Healthcare Research and Quality. Documentation for MEPS HC-163 2013 full year consolidated data file. 2013. Available at: http://meps.ahrq.gov/mepsweb/ data_stats/download_data/pufs/h163/h163doc.pdf Accessed July 26, 2018.

9. Karvellas CJ, Taylor S, Özelsel T, et al. A pilot randomized controlled trial of intra-operative continuous renal replacement therapy during liver transplantation: The inception study. Hepatology 2018;68:7A.

10. D'Hoore W, Bouckaert A, Tilquin C. Practical considerations on the use of the Charlson comorbidity index with administrative data bases. J Clin Epidemiol 1996;49:1429-1433.

11. Mahabaleshwarkar R, Khanna R, West-Strum D, et al. Association between health-related quality of life and colorectal cancer screening. Popul Health Manag 2013;16:178-189.

12. Li C, Ford ES, Mokdad AH, et al. Clustering of multiple healthy lifestyle habits and health-related quality of life among U.S. adults with diabetes. Diabetes Care 2007;30: 1770-1776.
13. Carlos RC, Fendrick AM, Patterson SK, et al. Associations in breast and colon cancer screening behavior in women. Acad Radiol 2005;12:451-458.

14. Hennessy CH, Moriarty DG, Zack MM, et al. Measuring health-related quality of life for public health surveillance. Public Health Rep 1994;109:665-672.

15. Ware J, Jr., Kosinski M, Keller SD. A 12-item short-form health survey: Construction of scales and preliminary tests of reliability and validity. Med Care 1996;34:220-233.

16. Brazier JE, Roberts J. The estimation of a preference-based measure of health from the SF-12. Med Care 2004;42:851-859.

17. Gray AM, Rivero-Arias O, Clarke PM. Estimating the association between SF-12 responses and EQ-5D utility values by response mapping. Med Decis Making 2006;26:18-29.

18. Shaw PA. The history of cervical screening I: The pap test. J SOGC 2000;22:110-114.

19. Siegel RL, Miller KD, Jemal A. Cancer statistics, 2019. CA Cancer J Clin 2019;69:7-34.

20. Chastek B, Harley C, Kallich J, et al. Health care costs for patients with cancer at the end of life. J Oncol Pract 2012;8: $75 \mathrm{~s}-80 \mathrm{~s}$.

21. Taplin SH, Barlow W, Urban N, et al. Stage, age, comorbidity, and direct costs of colon, prostate, and breast cancer care. J Natl Cancer Inst 1995;87:417-426.

22. Pfaendler KS, Wenzel L, Mechanic MB, Penner KR. Cervical cancer survivorship: long-term quality of life and social support. Clin Ther 2015;37:39-48.

23. Lin X-J, Lin IM, Fan S-Y. Methodological issues in measuring health-related quality of life. Tzu Chi Med J 2013;25:8-12.

24. Kent EE, Ambs A, Mitchell SA, et al. Health-related quality of life in older adult survivors of selected cancers: Data from the SEER-MHOS linked data resource. Cancer 2015;121:758-765.

25. Endarti D, Riewpaiboon A, Thavorncharoensap M, et al. Evaluation of health-related quality of life among patients with cervical cancer in Indonesia. Asian Pac J Cancer Prev 2015;16:3345-3350.

26. Korfage IJ, Essink-Bot ML, Mols F, et al. Health-related quality of life in cervical cancer survivors: A population-based survey. Int J Radiat Oncol Biol Phys 2009;73:1501-1509.

27. Yousefi M, Najafi S, Ghaffari S, et al. Comparison of SF6D and EQ-5D scores in patients with breast cancer. Iran Red Crescent Med J 2016;18:e23556.

28. Chen J, Wong CK, McGhee SM, et al. A comparison between the EQ-5D and the SF-6D in patients with chronic obstructive pulmonary disease (COPD). PLoS One 2014;9:e112389.

29. Bharmal M, Thomas J, 3rd. Comparing the EQ-5D and the SF-6D descriptive systems to assess their ceiling effects in the US general population. Value Health 2006;9:262-271.

30. Yabroff KR, Warren JL, Banthin J, et al. Comparison of approaches for estimating prevalence costs of care for cancer patients: What is the impact of data source. Med Care 2009;47(7 Suppl 1):S64-S69.

31. Abraham AG, D'Souza G, Jing Y, et al. Invasive cervical cancer risk among HIV-infected women: A North American multicohort collaboration prospective study. J Acquir Immune Defic Syndr 2013;62:405-413.

Address correspondence to: Ruchit Shah, PhD Pharmerit International 4350 East West Hwy, Suite 1110 Bethesda, MD 20814

E-mail: rshah@pharmerit.com 